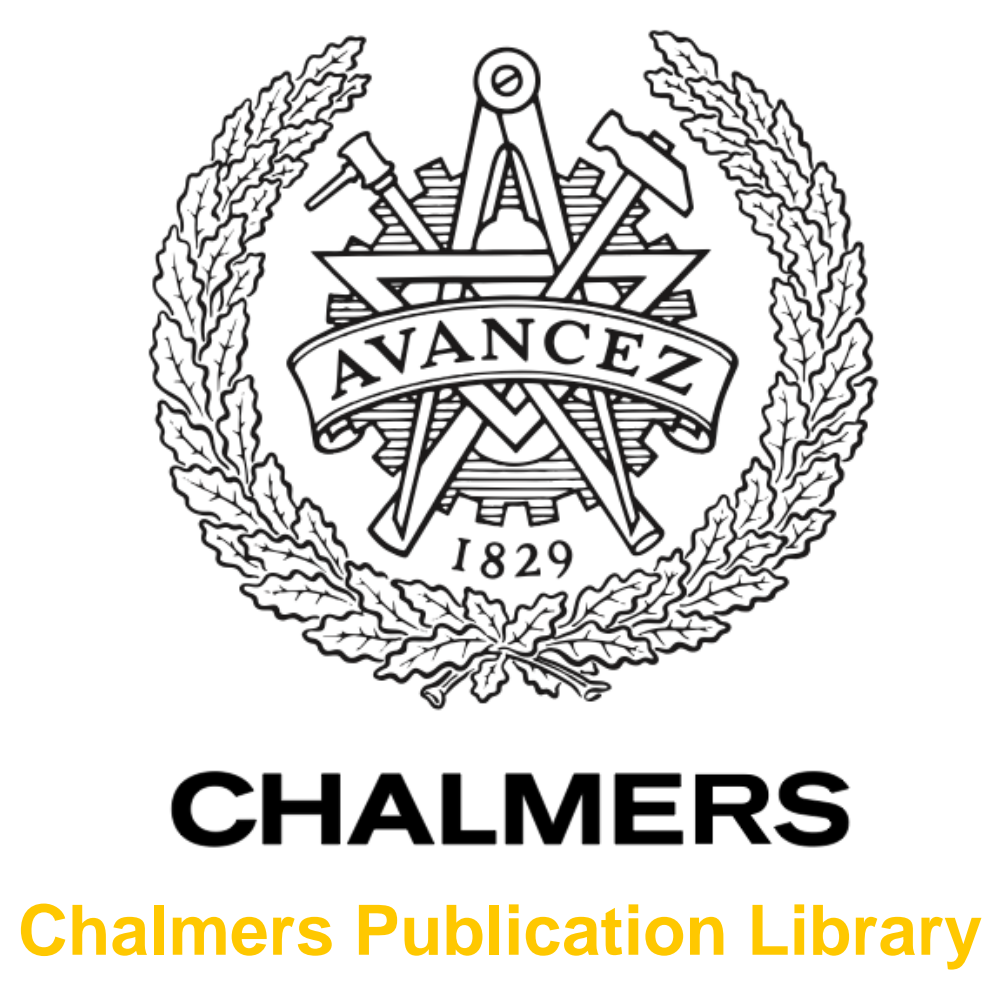

\title{
Array-fed cylindrical reflector antenna for automotive OTA tests in Random Line- Of-Sight
}

This document has been downloaded from Chalmers Publication Library (CPL). It is the author's version of a work that was accepted for publication in:

2016 10th European Conference on Antennas and Propagation, EuCAP 2016

\author{
Citation for the published paper: \\ Razavi, A. ; Glazunov, A. ; Kildal, P. et al. (2016) "Array-fed cylindrical reflector antenna \\ for automotive OTA tests in Random Line-Of-Sight". 2016 10th European Conference on \\ Antennas and Propagation, EuCAP 2016 pp. 7481689.
}

http://dx.doi.org/10.1109/EuCAP.2016.7481689

Downloaded from: http://publications.lib.chalmers.se/publication/246234

Notice: Changes introduced as a result of publishing processes such as copy-editing and formatting may not be reflected in this document. For a definitive version of this work, please refer to the published source. Please note that access to the published version might require a subscription.

Chalmers Publication Library (CPL) offers the possibility of retrieving research publications produced at Chalmers University of Technology. It covers all types of publications: articles, dissertations, licentiate theses, masters theses, conference papers, reports etc. Since 2006 it is the official tool for Chalmers official publication statistics. To ensure that Chalmers research results are disseminated as widely as possible, an Open Access Policy has been adopted.

The CPL service is administrated and maintained by Chalmers Library. 


\title{
Array-fed Cylindrical Reflector Antenna for Automotive OTA Tests in Random Line-Of-Sight
}

\author{
Aidin Razavi, Andrés Alayón Glazunov, Per-Simon Kildal, and Rob Maaskant \\ Dept. of Signals and Systems, Chalmers University of Technology, Gothenburg, Sweden \\ aidin.razavi@chalmers.se; andres.glazunov@chalmers.se; per-simon.kildal@chalmers.se; rob.maaskant@chalmers.se
}

\begin{abstract}
In this paper we look into a near-field measurement setup consisting of a cylindrical reflector and a linear array feed. The main application is Random Line-Of-Sight Over-TheAir measurements in anechoic or semi-anechoic chambers (i.e., anechoic chamber with reflecting floor). More specifically, the aim is testing the wireless communication systems on automotive vehicles. The aim is to generate the uniform field distribution of a plane wave over a pre-defined test zone containing the car or at least the part of the car containing the antenna(s). The field intensity variations over the testing zone have been investigated for reference measurements over the testing frequency bandwidth, i.e., $1.6-2.8$ GHz.
\end{abstract}

Index Terms-Random Line-Of-Sight, OTA measurement, Vehicular antennas.

\section{INTRODUCTION}

Autonomous driving cars have many potential advantages such as reduced traffic collisions, improved traffic flow management and increased roadway capacity. At the same time consumers require more and more connectivity in their vehicles for, e.g., infotainment systems, streaming music services, maps and satellite navigation systems. These systems require an increasingly higher number of wireless links to and from cars. The wireless connection must be reliable to ensure the service quality and passenger safety, especially in the case of the autonomous driving cars where safety is a primary concern. This brings about a need for Over-The-Air (OTA) testing of wireless communication to and from cars [1].

Generally, it is possible to perform OTA testing of wireless devices in both anechoic chambers (walls fitted with absorbers) and reverberation chambers (reflecting walls) [2], [3]. Anechoic chamber emulates the pure Line-Of-Sight (LOS) environment which traditionally is used for testing the performance of antennas in fixed installations, whereas the reverberation chamber represents the Rich Isotropic Multipath (RIMP) environment where large amounts of scattering are present, such as urban or indoor environments. For the automotive wireless applications, especially in areas with less density of scatterers, LOS is expected to play a more dominant role than RIMP in the environment. The same is true for millimeter wave $5 \mathrm{G}$ telecommunications where the path loss is larger and the spacing between the scatterers is large in terms of wavelength.

This work has been supported by two projects from Sweden's innovation agency VINNOVA, one within the VINN Excellence Center Chase at Chalmers and another via the program Innovative ICT 2013, and by internal support from Chalmers.
However, it should be noted that due to the randomness of the orientation of the cars, fixed LOS does not apply to the communication link and instead Random Line-Of-Sight (RLOS) has been introduced to describe the new OTA test scenario [4], [5].

In this paper, we provide an initial study of the chamber antenna for OTA test of the automotive wireless systems in anechoic chambers. The proposed antenna system consists of a cylindrical reflector which is fed with a linear array. The elements of the linear array are initially considered as Huygens sources, but will eventually be replaced by wideband bowtie elements [6], [7]. The goal of the design study is to produce a good plane wave in the test zone. The quality of this plane wave is characterized by the standard deviation of the variations of the field distribution within the test zone when the car is not present [8]. The test zone is defined by the location of the turntable on which the car will be located, and the location of the Antenna Under Test (AUT) relative to the axis of rotation as well as the AUT height. In the reference measurements, the AUT is a vertical monopole, whereas in the actual car measurements it will be the antenna on the car. We have performed a previous study with a linear array as the chamber antenna. This showed that by using 24 elements in the array, it is possible to achieve a standard deviation (STD) of the field distribution of almost $0.7 \mathrm{~dB}$ over a test zone of radius $1 \mathrm{~m}$ [8]. This radius was chosen to allow for a $1 \mathrm{~m}$ displacement of the AUT relative to the rotation axis. In another study we have used a $24 \times 24$ elements square array in order to emulate the plane wave in the testing zone and also to be able to model base station antennas' height and tilting [9]. Here, we employ a cylindrical reflector in conjunction with the linear array to achieve the same capabilities as the planar array. We use the ideal digital threshold receiver model [10] to determine the theoretical Probability of Detection (PoD) of the reference simulations, and this will be a vertical line if the field distribution is constant. In this work we limit the simulations to vertical polarization because most car antennas are located on the roof of the car and thereby can only receive vertically polarized waves.

\section{Field Simulations and Receiver Model}

The geometry of the reflector, the feed and the test zone are illustrated in Fig. 1. The reflector's focal distance, height and length are assumed to be $F=1.5 \mathrm{~m}, h_{\mathrm{r}}=2 F=3 \mathrm{~m}$ and $L_{\mathrm{r}}=$ 


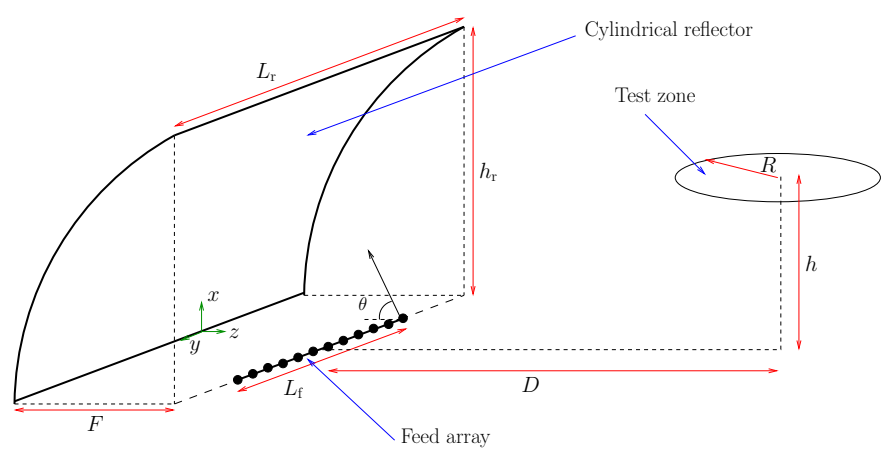

Fig. 1: Cylinndrical reflector, linear array feed and the test zone.

$4 \mathrm{~m}$, respectively. The length of the feed array is $L_{\mathrm{f}}$ which will be defined by the number of antenna elements and element spacing. The test zone is located at a $4 \mathrm{~m}$ distance from the feed and its radius and height are $R$ and $h$, respectively. In practice $R$ and $h$ are defined by the location of the AUT on the vehicle with respect to the turntable's axis. The feed is designed to have its main beam at an angle $\theta=55^{\circ}$ from the $-z$ direction while illuminating the reflector.

We have used Physical Optics (PO) to calculate the scattered field from the reflector. The feed is assumed to be an array of vertically polarized Huygens sources. Fig. 2 illustrates the intensity of the incident field from the source $\left(\boldsymbol{E}^{\mathrm{i}}\right)$, scattered field from the reflector $\left(\boldsymbol{E}^{\mathrm{s}}\right)$, and the total field $\left(\boldsymbol{E}^{\mathrm{t}}=\boldsymbol{E}^{\mathrm{i}}+\boldsymbol{E}^{\mathrm{s}}\right)$ over the $x z$-plane for a single element feed. As can be seen in this figure, the feed itself can have strong direct radiation into the test zone. This direct radiation from the feed has an adverse effect on the accuracy of the measurement. In fact, in the actual realization of the chamber antenna, we will choose array elements with more directive beams than Huygens sources, or block the direct radiation by a corrugated shield. For this reason, all the results in the current paper are based on the values of the scattered field $\boldsymbol{E}^{\mathrm{s}}$. Hence, basing the results on $\boldsymbol{E}^{\mathrm{s}}$ instead of $\boldsymbol{E}^{\mathrm{t}}$ is a practical assumption.

The receiver is modeled by the ideal threshold receiver model [10]. In this model the system throughput is given by

$$
\begin{aligned}
\text { TPUT } & =\operatorname{TPUT}_{\max }\left(1-\mathrm{CDF}\left(P_{\mathrm{th}} / P_{\mathrm{av}}\right)\right) \\
& =\operatorname{TPUT}_{\max } \operatorname{PoD}\left(P_{\mathrm{av}} / P_{\mathrm{th}}\right)
\end{aligned}
$$

where TPUT $_{\max }$ is the maximum system throughput, CDF is the Cumulative Distribution Function, PoD is the Probability of Detection, $P_{\mathrm{th}}$ is the threshold power level which can be determined by conducted measurements, and $P_{\mathrm{av}}$ is the available average power at the input of the threshold receiver.

\section{Figures OF MERIT}

We have chosen two figures of merit to characterize the accuracy of the chamber antenna. Both of these figures of merit are related to the variation of the field inside the test zone. As mentioned earlier, the location of the test zone depends on the placement of the AUT with respect to the axis of the rotating platform in the chamber. Based on typical size of
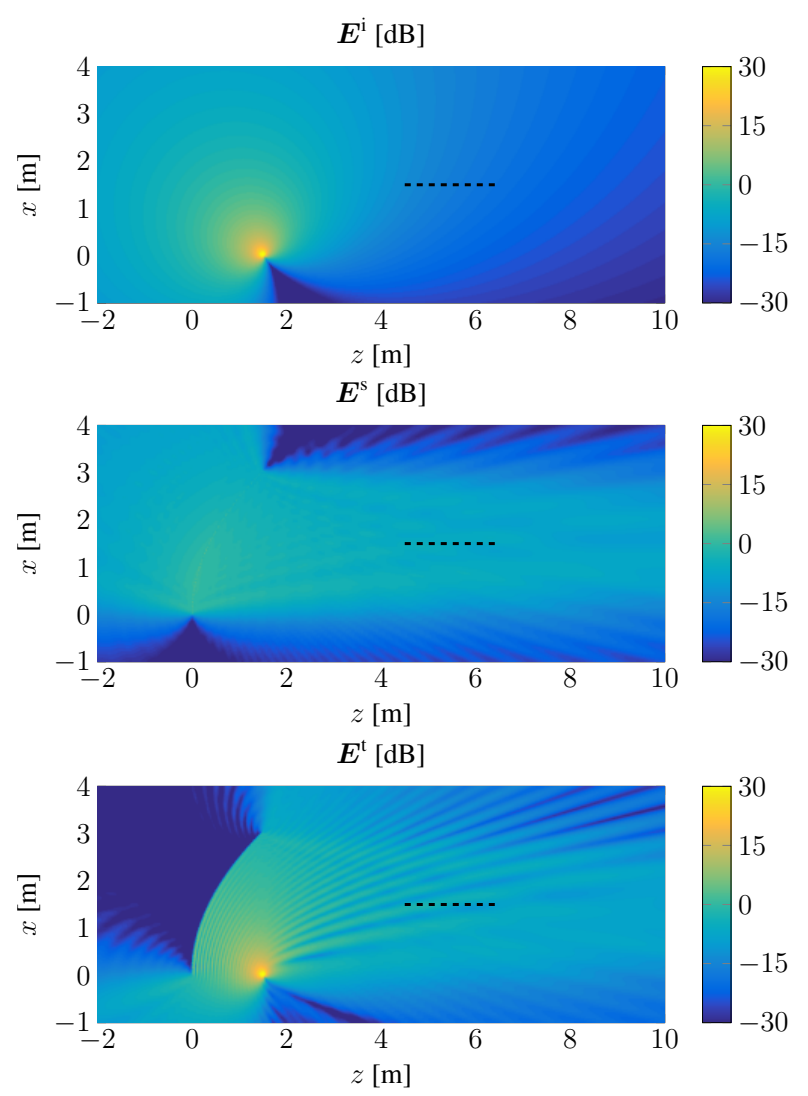

Fig. 2: The intensity of the incident, scattered and total electric fields at $2 \mathrm{GHz}$. The test zone is a circle in $z y$-plane and its side view is shown with the dashed line.

personal cars, we choose $R=1 \mathrm{~m}, h=1.5 \mathrm{~m}$, and $D=$ $4 \mathrm{~m}$. The first figure of merit is the standard deviation (STD) of the normalized received power at a vertical small dipole antenna moving over the surface of the test zone, in $\mathrm{dB}$ scale. Assuming a vertical polarization for the chamber antenna and the AUT, the normalized received power is computed as

$$
\frac{P_{\text {rec }}}{P_{\text {ref }}}=\frac{\left|E_{x}(\boldsymbol{r})\right|^{2}}{\frac{1}{\pi R^{2}} \int_{0}^{2 \pi} \int_{0}^{2 R}\left|E_{x}(\boldsymbol{r}(\rho, \phi))\right|^{2} \rho \mathrm{d} \rho \mathrm{d} \phi}
$$

where $E_{x}$ is the vertical component of the electric field.

The second figure of merit is the difference (in $\mathrm{dB}$ scale) between the received power and the threshold power at 0.9 PoD level, which corresponds to receiving $90 \%$ of the transmitted bitstream. Our target accuracy is $1 \mathrm{~dB}$ or less for both figures of merit.

\section{REsults}

We have used the same number of elements $\left(N_{\text {ant }}\right)$, element spacing $\left(d_{\mathrm{h}}\right)$, and amplitude tapering for the feed array as in [8]. That is, $N_{\text {ant }}=24, d_{\mathrm{h}}=130.9 \mathrm{~mm}$, and a linear-in-dB amplitude taper from 0 to $-6 \mathrm{~dB}$ on $25 \%$ of the elements on each side of the array. The scattered field $\boldsymbol{E}^{\mathrm{s}}$ at the middle of the reflector's height $(h=1.5 \mathrm{~m})$ and the corresponding PoD curves for various values of $R$ between 0 and $1 \mathrm{~m}$ are 

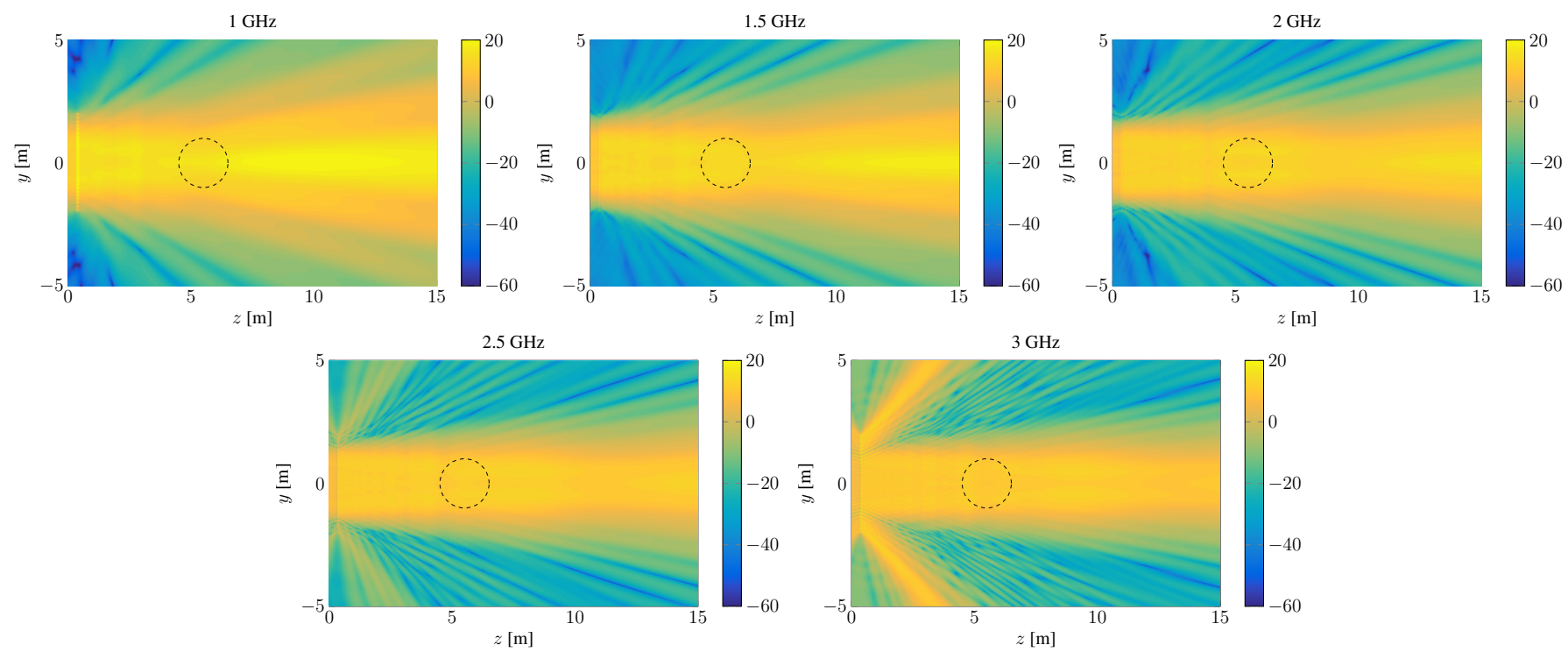

Fig. 3: The scattered field $\boldsymbol{E}^{\mathrm{s}}$ at the middle of the reflector's height $(h=1.5 \mathrm{~m}$ ) at different frequencies. The test zone is shown with the dashed black circle.
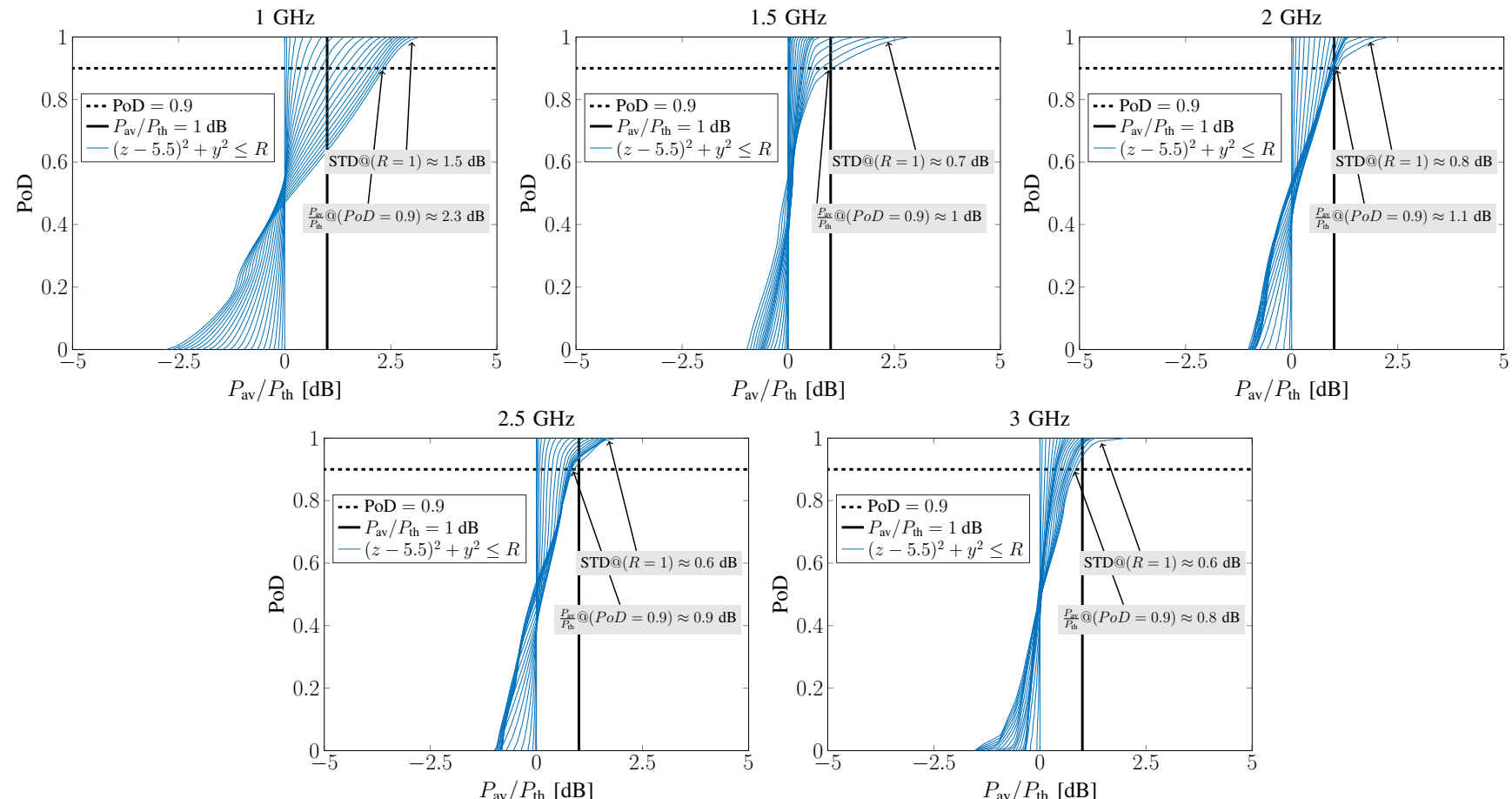

Fig. 4: PoD curves for various values of $R$ between 0 and $1 \mathrm{~m}$ in the test zone at different frequencies.

shown in Fig. 3 and 4 for several frequencies, respectively. As can be seen from Fig. 3, grating lobes appear at higher frequency. On the other hand, it is shown in Fig. 4 that more homogeneous field distribution is obtained in the testing zone as the frequency increases. As the field distribution in the testing zone becomes more homogeneous, the PoD curves get closer to the vertical line at $0 \mathrm{~dB}$ which is the ideal threshold level when the plane wave has constant amplitude over the test zone.
We have compared the performance of the array-fed cylindrical reflector, with that of a $24 \times 24$ elements square array of comparable size and same vertical and horizontal element spacing, and the same height as the cylindrical reflector. Two different amplitude tapering schemes are used on the planar array: Horizontal taper denotes the case where tapering is applied only in the horizontal array dimension. Horizontal and Vertical (2D) taper indicates the case where the same tapering is applied in both array dimensions. Fig. 5 shows the 

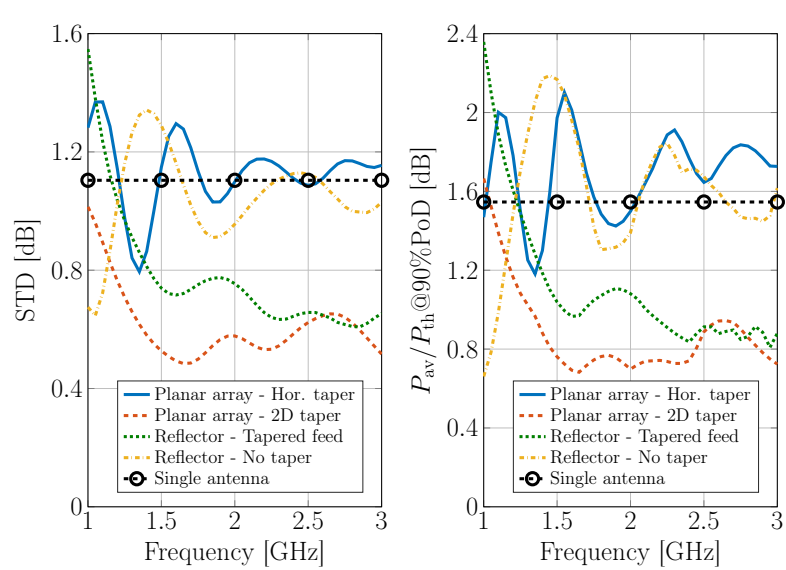

Fig. 5: The two figures of merit vs. frequncy for Cylindrical reflector compared with those of planar arrays with two different amplitude tapering schemes and a single antenna.

two figures of merit over the frequency band $1-3 \mathrm{GHz}$ for the cylindrical reflector with both tapered and uniform feeds. The values are compared to those of the horizontally-tapered planar array, and the 2D-tapered planar array. The case of a single antenna is also plotted in the same figure for comparison. It can be seen that the reflector with a non-tapered feed provides an uncertainty similar to that of a planar array with horizontal taper. Also, the single antenna has a similar uncertainty as the reflector with a non-tapered feed. Furthermore, the reflector with tapered feed has an accuracy comparable to that of the 2D-tapered planar array. But, the reflector with linear array feed employs much smaller number of antenna elements. Hence, a less complex and more practical feeding network is required for the array structure. As shown in Fig. 5, the use of a cyclindrical reflector and linear array feed can provide smaller than $0.8 \mathrm{~dB}$ STD values and smaller than almost $1 \mathrm{~dB}$ values of power at $90 \%$ PoD level, at frequencies above $1.5 \mathrm{GHz}$. For frequencies below $1.5 \mathrm{GHz}$, the size of the reflector is not large enough to radiate homogeneous field distribution at the distance of the testing zone.

Another factor which affects the accuracy levels and the figures of merit over the test zone is elevation. The elevation of the AUT measured from the floor of the measurement chamber will depend on the size and shape of the vehicle. The height of personal cars typically varies between 1.5 and $1.8 \mathrm{~m}$. Hence, it is interesting to investigate the accuracy of the chamber antenna at horizontal planes with different heights. To this end, Fig. 6 shows the two figures of merit for the cylindrical reflector, over the frequency band, for different elevations from $1.5 \mathrm{~m}$ to $1.8 \mathrm{~m}$. It can be observed in this figure that variations in elevation can lead to around $0.4 \mathrm{~dB}$ change in the figures of merit. However, the effect of the elevation on the uncertainty levels is complex and requires further investigation.

\section{Conclusions}

A setup consisting of a cylindrical reflector and a linear array feed is proposed as the chamber antenna for RLOS automotive OTA measurements in anechoic chambers. The
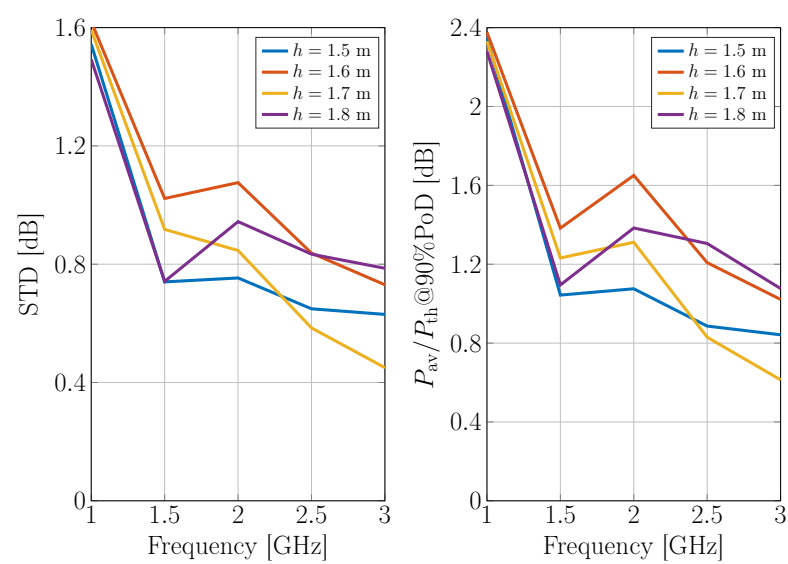

Fig. 6: The two figures of merit vs. frequncy for cylindrical reflector. The test zone is located at different elevations between $1.5 \mathrm{~m}$ to $1.8 \mathrm{~m}$.

goal is to generate a uniform plane wave field distribution over a circular testing zone, inside which the antenna on the car will be located. Two figures of merit are used to measure the accuracy. It is shown that values below $1 \mathrm{~dB}$ are achievable for both figures of merit over the desired frequency band, i.e., 1.6$2.8 \mathrm{GHz}$. The achieved accuracy levels with only 24 antenna elements in the feed are comparable to those of a large planar array with $24 \times 24$ elements.

\section{REFERENCES}

[1] P.-S. Kildal, A. A. Glazunov, J. Carlsson, and A. Majidzadeh, "Costeffective measurement setups for testing wireless communication to vehicles in reverberation chambers and anechoic chambers," in Antenna Measurements \& Applications (CAMA), 2014 IEEE Conference on. IEEE, 2014, pp. 1-4.

[2] A. A. Glazunov, V.-M. Kolmonen, and T. Laitinen, "MIMO over-theair testing," LTE-Advanced and Next Generation Wireless Networks: Channel Modelling and Propagation, pp. 411-441, 2012.

[3] P.-S. Kildal, C. Orlenius, and J. Carlsson, "OTA testing in multipath of antennas and wireless devices with MIMO and OFDM," Proceedings of the IEEE, vol. 100, no. 7, pp. 2145-2157, 2012.

[4] P. Kildal and J. Carlsson, "New approach to OTA testing: RIMP and pure-LOS reference environments \& a hypothesis," in Antennas and Propagation (EuCAP), 2013 7th European Conference on. IEEE, 2013, pp. 315-318.

[5] P.-S. Kildal, "Rethinking the wireless channel for OTA testing and network optimization by including user statistics: RIMP, pure-LOS, throughput and detection probability," in 2013 Proceedings of the International Symposium on Antennas and Propagation (ISAP), Vols 1 and 2, vol. 1, 2013.

[6] H. Raza, A. Hussain, J. Yang, and P.-S. Kildal, "Wideband compact 4-port dual polarized self-grounded bowtie antenna," Antennas and Propagation, IEEE Transactions on, vol. 62, no. 9, pp. 4468-4473, 2014.

[7] P.-S. Kildal, X. Chen, M. Gustafsson, and Z. Shen, "Mimo characteri zation on system level of $5 \mathrm{~g}$ microbase stations subject to randomness in los," Access, IEEE, vol. 2, pp. 1064-1077, 2014.

[8] A. Alayón Glazunov, P.-S. Kildal, and M. Schilliger Kildal, "Devising a horizontal chamber array for automotive OTA tests in random lineof-sight," in Internationl Symposium on Antennas And propagation (ISAP2015), 2015.

[9] A. Alayón Glazunov, A. Razavi, and P.-S. Kildal, "Simulations of a planar array arrangement for automotive random-los ota testing," (submitted to EuCAP2016).

[10] P.-S. Kildal, A. Hussain, X. Chen, C. Orlenius, A. Skårbratt, J. Åsberg, T. Svensson, and T. Eriksson, "Threshold receiver model for throughput of wireless devices with MIMO and frequency diversity measured in reverberation chamber," Antennas and Wireless Propagation Letters, IEEE, vol. 10, pp. 1201-1204, 2011. 